PHYSICAL REVIEW D 92, 021101(R) (2015)

\title{
New solar axion search using the CERN Axion Solar Telescope with ${ }^{4}$ He filling
}

M. Arik, ${ }^{1, *}$ S. Aune, ${ }^{2}$ K. Barth, ${ }^{3}$ A. Belov, ${ }^{4}$ H. Bräuninger, ${ }^{5}$ J. Bremer, ${ }^{3}$ V. Burwitz, ${ }^{5}$ G. Cantatore,${ }^{6}$ J. M. Carmona, ${ }^{7}$ S. A. Cetin, ${ }^{1}$ J. I. Collar, ${ }^{8}$ E. Da Riva, ${ }^{3}$ T. Dafni, ${ }^{7}$ M. Davenport, ${ }^{3}$ A. Dermenev, ${ }^{4}$ C. Eleftheriadis, ${ }^{9}$ N. Elias, ${ }^{3, \dagger}$ G. Fanourakis, ${ }^{10}$ E. Ferrer-Ribas, ${ }^{2}$ J. Galán, ${ }^{2}$ J. A. García, ${ }^{7, \$}$ A. Gardikiotis, ${ }^{11}$ J. G. Garza, ${ }^{7}$ E. N. Gazis, ${ }^{12}$ T. Geralis, ${ }^{10}$ E. Georgiopoulou, ${ }^{11}$ I. Giomataris, ${ }^{2}$ S. Gninenko, ${ }^{4}$ M. Gómez Marzoa, ${ }^{3,8}$ M. D. Hasinoff, ${ }^{13}$ D. H. H. Hoffmann, ${ }^{14}$ F. J. Iguaz, ${ }^{7}$ I. G. Irastorza, ${ }^{7}$ J. Jacoby, ${ }^{15}$ K. Jakovčić,,${ }^{16}$ M. Karuza, ${ }^{6,17}$ M. Kavuk, ${ }^{1, \|}$ M. Krčmarar, ${ }^{16}$ M. Kuster, ${ }^{5,14,9}$ B. Lakić, ${ }^{16}$ J. M. Laurent, ${ }^{3}$ A. Liolios, ${ }^{9}$ A. Ljubičić, ${ }^{16}$ G. Luzón, ${ }^{7}$ S. Neff, ${ }^{14}$ T. Niinikoski, ${ }^{3, * *}$ A. Nordt, ${ }^{5,14, \dagger}$ I. Ortega, ${ }^{7,3}$ T. Papaevangelou, ${ }^{2}$ M. J. Pivovaroff, ${ }^{18}$ G. Raffelt, ${ }^{19}$ A. Rodríguez, ${ }^{7}$ M. Rosu, ${ }^{14}$ J. Ruz, ${ }^{18}$ I. Savvidis, ${ }^{9}$ I. Shilon, ${ }^{7,3+*}$ S. K. Solanki, ${ }^{20,8}$ L. Stewart, ${ }^{3}$ A. Tomás, ${ }^{7}$ T. Vafeiadis, ${ }^{3,9,11}$ J. Villar, ${ }^{7}$ J. K. Vogel, ${ }^{18}$ S. C. Yildiz, ${ }^{1,\|\|}$ and K. Zioutas ${ }^{3,11}$

(CAST Collaboration)

\author{
${ }^{1}$ Dogus University, Istanbul, Turkey \\ ${ }^{2}$ IRFU, Centre d'Études Nucléaires de Saclay (CEA-Saclay), Gif-sur-Yvette, France \\ ${ }^{3}$ European Organization for Nuclear Research (CERN), Genève, Switzerland \\ ${ }^{4}$ Institute for Nuclear Research (INR), Russian Academy of Sciences, Moscow, Russia \\ ${ }^{5}$ Max-Planck-Institut für Extraterrestrische Physik, Garching, Germany \\ ${ }^{6}$ Istituto Nazionale di Fisica Nucleare (INFN), Sezione di Trieste and Università di Trieste, Trieste, Italy \\ ${ }^{7}$ Grupo de Investigación de Física Nuclear y Astropartículas, Universidad de Zaragoza, Zaragoza, Spain \\ ${ }^{8}$ Enrico Fermi Institute and KICP, University of Chicago, Chicago, Illinois 60637, USA \\ ${ }^{9}$ Aristotle University of Thessaloniki, Thessaloniki, Greece \\ ${ }^{10}$ National Center for Scientific Research “Demokritos", Athens, Greece \\ ${ }^{11}$ Physics Department, University of Patras, Patras, Greece \\ ${ }^{12}$ National Technical University of Athens, Athens, Greece \\ ${ }^{13}$ Department of Physics and Astronomy, University of British Columbia, Vancouver, Canada \\ ${ }^{14}$ Technische Universität Darmstadt, IKP, Darmstadt, Germany \\ ${ }^{15}$ Johann Wolfgang Goethe-Universität, Institut für Angewandte Physik, Frankfurt am Main, Germany \\ ${ }^{16}$ Rudjer Bošković Institute, Zagreb, Croatia \\ ${ }^{17}$ Physics Department and Center for Micro and Nano Sciences and Technologies, University of Rijeka, \\ Radmile Matejcic 2, 51000 Rijeka, Croatia \\ ${ }^{18}$ Lawrence Livermore National Laboratory, Livermore, California 94550, USA \\ ${ }^{19}$ Max-Planck-Institut für Physik (Werner-Heisenberg-Institut), München, Germany \\ ${ }^{20}$ Max-Planck-Institut für Sonnensystemforschung, Göttingen, Germany
}

(Received 3 March 2015; published 28 July 2015)

The CERN Axion Solar Telescope (CAST) searches for $a \rightarrow \gamma$ conversion in the 9 T magnetic field of a refurbished LHC test magnet that can be directed toward the Sun. Two parallel magnet bores can be filled with helium of adjustable pressure to match the x-ray refractive mass $m_{\gamma}$ to the axion search mass $m_{a}$. After the vacuum phase (2003-2004), which is optimal for $m_{a} \lesssim 0.02 \mathrm{eV}$, we used ${ }^{4} \mathrm{He}$ in 2005-2007 to cover the mass range of $0.02-0.39 \mathrm{eV}$ and ${ }^{3} \mathrm{He}$ in $2009-2011$ to scan from 0.39 to $1.17 \mathrm{eV}$. After improving the

\footnotetext{
*Present address: Bogazici University, Istanbul, Turkey.

${ }^{\dagger}$ Present address: European Spallation Source ESS AB, Lund, Sweden.

Corresponding author.

jagarpas@unizar.es

${ }_{\S}^{\S}$ Also at: Laboratoire de Transfert de Chaleur et de Masse, École Polytechnique Fédérale de Lausanne (EPFL), Lausanne, Switzerland.

"Present address: Bogazici University, Istanbul, Turkey.

Present address: European XFEL GmbH, Notkestrasse 85, 22607 Hamburg, Germany.

***esent address: Excellence Cluster Universe, Technische Universität München, Garching, Germany.

†Present address: European Spallation Source ESS AB, Lund, Sweden.

\#Also at: Physics Department, Ben-Gurion University of the Negev, Beer-Sheva 84105, Israel.

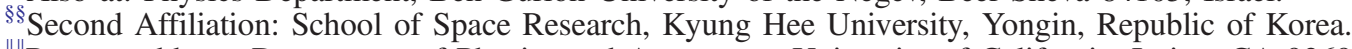

"I\| Present address: Department of Physics and Astronomy, University of California, Irvine, CA 92697, USA.
}

Published by the American Physical Society under the terms of the Creative Commons Attribution 3.0 License. Further distribution of this work must maintain attribution to the author(s) and the published article's title, journal citation, and DOI. 
detectors and shielding, we returned to ${ }^{4} \mathrm{He}$ in 2012 to investigate a narrow $m_{a}$ range around $0.2 \mathrm{eV}$ ("candidate setting" of our earlier search) and 0.39-0.42 eV, the upper axion mass range reachable with ${ }^{4} \mathrm{He}$, to "cross the axion line" for the KSVZ model. We have improved the limit on the axion-photon coupling to $g_{a \gamma}<1.47 \times 10^{-10} \mathrm{GeV}^{-1}$ (95\% C.L.), depending on the pressure settings. Since 2013, we have returned to the vacuum and aim for a significant increase in sensitivity.

DOI: 10.1103/PhysRevD.92.021101

\section{INTRODUCTION}

The low-energy frontier of elementary particle physics [1-6] includes numerous experimental efforts, ranging from the search for neutrinoless double beta decay and searches for nucleon and electron electric dipole moments all the way to searches for new low-mass bosons. The best-motivated case for the latter remains the axion, the pseudo-NambuGoldstone boson of a new broken global $U(1)$ symmetry, that is required in the context of the Peccei-Quinn mechanism to explain why $C P$-violating effects are extremely small or absent in QCD $[7,8]$. The axion is also an excellent candidate for the cold dark matter of the universe [9] and ongoing experimental searches as well as new efforts have recently gained fresh momentum [10-23]. On the other hand, the phenomenology of axions has inspired the proposition of axionlike particles (ALPs) and other WISPs (weakly interacting sub-eV particles) with additional theoretical motivations from string theory and cosmology. Experimental searches for low-mass bosons include precision searches for new long-range forces [24-27] and oscillating nucleon electric dipole moments [28-30].

The most promising technique to search for axions to date remains Sikivie's idea to use the two-photon vertex of axions or ALPs and look for their conversion to photons in a strong external magnetic field $B$ (also referred to as the Primakoff effect) [31]. The smallness of the coupling strength $g_{a \gamma}$ can be overcome by coherent conversion in a macroscopic volume. For more than ten years, the CERN Axion Solar Telescope (CAST) has pursued this idea in the form of a large axion helioscope [32-36]. Earlier helioscope searches were conducted in Brookhaven [37] and Tokyo [38-40]. In CAST, a refurbished LHC dipole magnet (length $9.26 \mathrm{~m}$, field strength $9.0 \mathrm{~T}$ ) is mounted to follow the Sun for approximately $1.5 \mathrm{~h}$ at both sunrise and sunset. In this way various x-ray detectors at both ends of the magnet are used to look at the Sun.

For a fixed axion-photon coupling strength $g_{a \gamma}$, the $a-\gamma$ conversion probability decreases when the $a-\gamma$ oscillation length becomes smaller than the magnet length, which limits CAST's sensitivity to an axion mass range $m_{a} \lesssim 0.02 \mathrm{eV}$. For larger $m_{a}$ values, the transition rate can be restored by providing photons with a refractive mass using a low- $Z$ gas filling [41]. The gas density (i.e. pressure) defines the search mass and one needs to step through many pressure settings to search a broad $m_{a}$ range.

CAST has taken data since 2003 and produced the exclusion plot shown in Fig. 1. The vacuum phase
PACS numbers: 95.35.+d, 07.85.Nc, 14.80.Va, 84.71.Ba

(2003-2004) achieved the best sensitivity up to $m_{a} \sim$ $0.02 \mathrm{eV}$ because of the long exposure time. With ${ }^{4} \mathrm{He}$ filling (2005-2007), the maximum search mass was extended to $\sim 0.4 \mathrm{eV}$, corresponding to the ${ }^{4} \mathrm{He}$ vapor pressure at cryogenic temperatures. With ${ }^{3} \mathrm{He}$ in 2009 2011 we finally reached $1.17 \mathrm{eV}$, which exceeds the cosmological hot dark matter limit for axions [42-47]. For $m_{a}>0.6 \mathrm{eV}$ our ${ }^{3} \mathrm{He}$ search has reached the "axion line" for a typical hadronic axion model $[48,49](\mathrm{E} / \mathrm{N}=0$, see Fig. 1), i.e., the locus of $g_{a \gamma}$ vs $m_{a}$ which is motivated by QCD axions, as opposed to more general ALPs.

To make the experimental progress pushing the sensitivity to lower coupling constants of Fig. 1, a much larger helioscope, the International Axion Observatory (IAXO) [53-55], has been envisioned and, for very small $m_{a}$, a large-scale photon-regeneration experiment ALPS-II [56] has been put forward. Until these next-generation projects

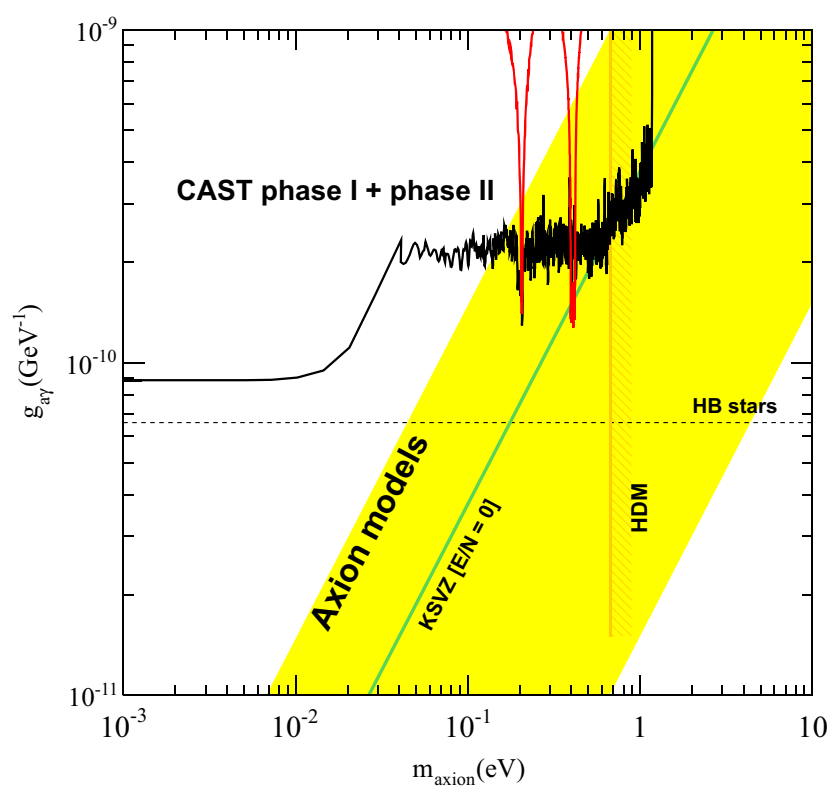

FIG. 1 (color online). Exclusion regions in the $m_{a}-g_{a \gamma}$ plane achieved by CAST in the vacuum phase $[32,33]$ and with ${ }^{4} \mathrm{He}$ [34] and ${ }^{3} \mathrm{He}[35,36]$ filling. We also show constraints from horizontal branch (HB) stars [50,51] (a similar limit stems from blue-loop suppression in massive stars [52]), and the hot dark matter (HDM) bound [42-47]. The yellow band represents typical theoretical models with $|E / N-1.95|=0.07-7$. The green solid line corresponds to $E / N=0$ as in the KSVZ model $[48,49]$, a typical hadronic axion model. In red we show our new limits near $m_{a}=0.2$ and $0.4 \mathrm{eV}$ from our 2012 data-taking campaign with ${ }^{4} \mathrm{He}$ gas. 
become operational, CAST can push its boundaries by reducing background levels and increasing the exposure time. Therefore, CAST has engaged in a program of technical improvements and renewed data taking to achieve these goals.

We report here on results from our 2012 data-taking campaign with ${ }^{4} \mathrm{He}$ gas. At each pressure setting, we integrated for about $7.5 \mathrm{~h}$ per detector (five solar trackings), in contrast to $1.5 \mathrm{~h}$ (one solar tracking) in our earlier ${ }^{4} \mathrm{He}$ campaign. On the sunset side, new Micromegas detectors with improved shielding and veto were installed, decreasing the background level by an approximate factor of 4 [57]. The search masses were selected to cover a previous "candidate setting" ( $\left.m_{a} \sim 0.2 \mathrm{eV}\right)$, where unusually many events above background had appeared, and the upper end of what can be reached with ${ }^{4} \mathrm{He}\left(m_{a} \sim 0.4 \mathrm{eV}\right)$ in order to reach the KSVZ (Kim, Shifman, Vainshtein, Zakharov model) axion line.

\section{SYSTEM DESCRIPTION}

After completing the ${ }^{3} \mathrm{He}$ phase, the gas was removed from the system. The magnet bores were then filled with ${ }^{4} \mathrm{He}$ to take advantage of the sophisticated gas metering system capable of filling the cold bore in small steps with a reproducibility of better than $100 \mathrm{ppm}$ [35].

As explained in Ref. [36], at increasingly higher gas densities (14-108 mbar at $1.8 \mathrm{~K}),{ }^{3} \mathrm{He}$ gas dynamics (convection and buoyancy) at the ends of the cold bore affect the density distribution along the cold bore. This effect progressively shortens the region with uniform density (effective coherence length) and also causes variations in the central density during the tilting of the magnet. In the ${ }^{4} \mathrm{He}$ case, the densities involved are relatively low (up to $15.5 \mathrm{mbar}$ at $1.8 \mathrm{~K}$ ) and these effects are negligible.

For ${ }^{4} \mathrm{He}$, the central gas density was calculated from the equation of state of the ${ }^{4} \mathrm{He}$ gas using the measured cold bore temperature, gas pressure and magnet vertical angle. The coherence length was taken as the magnet length of $9.26 \mathrm{~m}$.

While computational fluid dynamics (CFD) simulations have not been performed for ${ }^{4} \mathrm{He}$, the ${ }^{3} \mathrm{He}$ CFD simulations at a similar pressure over various vertical angles gave effective coherence lengths above $L_{\text {eff }}=8 \mathrm{~m}$. The main source of systematic error in the present result, according to our previous studies $[33,34,36]$ is given by the uncertainties on the gas dynamics inside the magnet bores. In order to estimate the effect of such uncertainties the upper limit calculation has been redone using a reduced effective coherence length $L_{\text {eff }}=8 \mathrm{~m}$ for all vertical angles. This value represents a worst case scenario of the effect of gas dynamics on $L_{\text {eff }}$ according to CFD simulations performed in Ref. [36].

The x-ray detectors installed at CAST during the 2012 data-taking campaign were three Micromegas detectors of the microbulk type [58-61] (one in the sunrise and two in the sunset side) and a pn-CCD detector in the focal plane of an X-ray telescope [62] on the sunrise side. While the detectors on the sunrise side remained unchanged since the previous data-taking campaign, the Micromegas detectors on the sunset side were upgraded, improving the background levels of the detectors. This is the result of low background techniques developed for the Micromegas detectors [57], where different strategies were exploited: the manufacturing technology of the novel microbulk Micromegas, the intrinsic radiopurity of the detectors [63], the discrimination algorithms on the analysis, and different shielding strategies.

The upgrade focuses on reducing the contribution of the environmental gamma flux. The lead shielding thickness was increased (from 25 to $100 \mathrm{~mm}$ ) and the design is more compact, improving the shielding around the pipes to the magnet. The inner copper shielding was increased from 5 to $10 \mathrm{~mm}$ of copper. It is connected to the magnet bores by a $10 \mathrm{~mm}$ thick copper pipe, which has an inner polytetrafluoroethylene coating of $2.5 \mathrm{~mm}$ thickness in order to attenuate the $8 \mathrm{keV}$ copper fluorescence. In addition, the aluminum strongback was replaced by a more radiopure copper one, and all the components close to the detector have been carefully selected and cleaned. A plastic scintillator was installed on the top of the shielding, allowing the discrimination of background events induced by cosmic muons in the detectors (see Ref. [57] for more details).

These upgrades reduce the background level to 1.3 and $1.7 \times 10^{-6} \mathrm{c} \mathrm{cm}^{-2} \mathrm{keV}^{-1} \mathrm{~s}^{-1}$ for the sunset detectors 1 and 2, respectively, i.e., about a factor 4 lower than before, improving the signal-to-noise ratio by a factor of 2 . Typical tracking and background spectra of one of the detectors in the sunset side are shown in Fig. 2. As seen both levels are compatible within their error. The background is dominated by the copper fluorescence at $8 \mathrm{keV}$, its escape peak above $5 \mathrm{keV}$ and the Argon fluorescence around $3 \mathrm{keV}$. According to our current understanding of

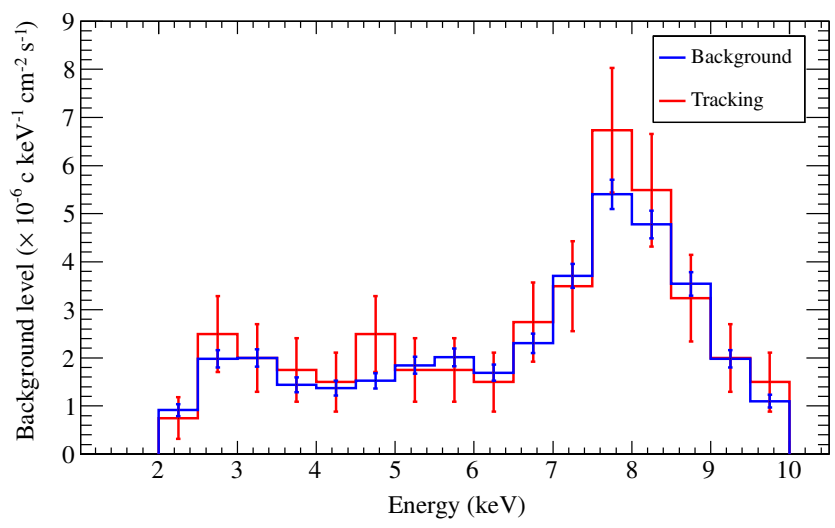

FIG. 2 (color online). Comparison between background (blue bars) and tracking (red bars) spectra of one Micromegas detectors installed in the sunset side. 
the background $[57,64,65]$, these fluorescences are induced by secondary particles generated in the inner materials by external radiation, mainly muons that are not tagged by the active veto system. On the other hand, the detector response has been fully characterized by a detailed simulation [65] and calibrations at different energies in an x-ray beam [66]. Moreover, daily calibrations allow us to monitor the performance of the detectors during the data-taking period (see Ref. [57] for more details).

The extensive research program for the background reduction continues; different measurements in special set-ups have been performed, such as the ones underground at the Laboratorio Subterráneo de Canfranc [67], where the cosmic muons are highly suppressed, and the test at surface level [66]. These special test benches, together with the simulations [65], were crucial in order to understand the origin of the background in the Micromegas detectors. Additional upgrades have been implemented at CAST for the rescanned vacuum phase that started in 2013, like the AFTER [68] front end electronics and a new design of the active shielding on the sunset side, with improved background levels on the Micromegas detectors further.

\section{DATA ANALYSIS AND RESULTS}

The results presented in this work are related to the data acquired with three Micromegas detectors during 2012 using ${ }^{4} \mathrm{He}$ as a buffer gas. The total exposure time in axionsensitive conditions (solar tracking) was about $147 \mathrm{~h}$ per detector with a background time of $2277 \mathrm{~h}$ per detector. In the first part of the 2012 data-taking campaign, the axion mass range $0.39<m_{a}<0.42 \mathrm{eV}$ was scanned, which corresponds to the pressure range $13.9-15.5$ mbar at $1.8 \mathrm{~K}$. During this period, 17 pressure settings were covered with a step size of $0.1 \mathrm{mbar}$ and an effective average exposure time of $\sim 24 \mathrm{~h}$ per setting. In the second part of the data-taking campaign, a single setting at $m_{a} \simeq 0.20 \mathrm{eV}$ was covered during $\sim 30 \mathrm{~h}$.

The analysis of the data was performed in the same manner as in our previous publications $[35,36]$. In order to take into account the variations of the density inside the magnet bores during the trackings, an unbinned likelihood was implemented, where the likelihood function can be expressed as

$$
\log \mathcal{L} \propto-R_{T}+\sum_{i}^{n} \log R\left(t_{i}, E_{i}, d_{i}\right)
$$

Here, $R_{T}$ is the expected number of counts from the axion to photon conversion over all the exposure time, energy and detectors. The sum is over each of the $n$ detected counts during the tracking time, for an expected rate $R\left(t_{i}, E_{i}, d_{i}\right)$ as a function of the event time $t_{i}$, energy $E_{i}$ and detector $d_{i}$, given by the expression

$$
R(t, E, d)=B_{d}+S(t, E, d),
$$

where $B_{d}$ is the background level of the detector $d$. $S(t, E, d)$ is the expected rate from axion conversion in the detector $d$ given by

$$
S(t, E, d)=\frac{d \Phi_{a}}{d E} P_{a \rightarrow \gamma} \epsilon_{d}
$$

Here, $\epsilon_{d}$ is the detector efficiency, $\frac{d \Phi_{a}}{d E}$ is the differential solar axion flux, which can be parametrized [50] by the expression

$$
\frac{d \Phi_{a}}{d E}=6.02 \times 10^{10} g_{10}^{2} \frac{E^{2.481}}{e^{E / 1.205}}\left[\mathrm{~cm}^{-2} \mathrm{~s}^{-1} \mathrm{keV}^{-1}\right]
$$

with $g_{10}=g_{a \gamma} /\left(10^{-10} \mathrm{GeV}^{-1}\right)$ and energies in $\mathrm{keV}$. $P_{a \rightarrow \gamma}$ is the axion to photon conversion probability inside a strong magnetic field [41], given by

$$
P_{a \rightarrow \gamma}=\left(\frac{g_{a \gamma} B}{2}\right)^{2} \frac{1+e^{-\Gamma L}-2 e^{-\Gamma L / 2} \cos (q L)}{q^{2}+\Gamma^{2} / 4},
$$

where $q=\left|m_{a}^{2}-m_{\gamma}^{2}\right| /(2 E)$ is the axion-photon momentum transfer in a magnetic field $B$ and $\Gamma$ is the absorption coefficient in the buffer gas.

As explained in Ref. [34], the dependence on $m_{a}$ in the expressions above is included in the conversion probability given in Eq. (5), where $P_{a \rightarrow \gamma}$ is enhanced for axion masses that match the refractive photon mass $m_{\gamma}$ as determined by the buffer gas density. Therefore for a given axion mass $m_{a}$, only the counts for which the coherence condition is fulfilled will contribute to the likelihood function $\log \mathcal{L}$.

By maximizing $\log \mathcal{L}$ a best-fit value $g_{\min }^{4}$ is obtained. This value is compatible with the absence of a signal in the entire axion mass range and thus an upper limit on $g_{a \gamma}$ is extracted, by integrating the Bayesian posterior probability from zero up to $95 \%$ with a flat prior in $g_{a \gamma}^{4}$. The computed upper limit for several values of $m_{a}$ is displayed in red in Fig. 1. A close view of the excluded region is shown in Fig. 3, where only the axion mass range scanned during the 2012 data-taking campaign is included.

This demonstrates that CAST improves its previous limit for axion masses $0.39<m_{a}<0.42 \mathrm{eV}$, down to an average value of the coupling constant $g_{a \gamma} \sim$ $1.47 \times 10^{-10} \mathrm{GeV}^{-1}$. Moreover, the "candidate setting" around $0.2 \mathrm{eV}$ was rescanned and in the absence of an axion signature a coupling limit of $g_{a \gamma}<1.40 \times 10^{-10} \mathrm{GeV}^{-1}$ at a $95 \%$ of C.L. was derived. In contrast to the previous results [34-36], the structure of the excluded region has a smooth shape, because of considerably larger exposure time per step, resulting in smaller statistical fluctuations. 

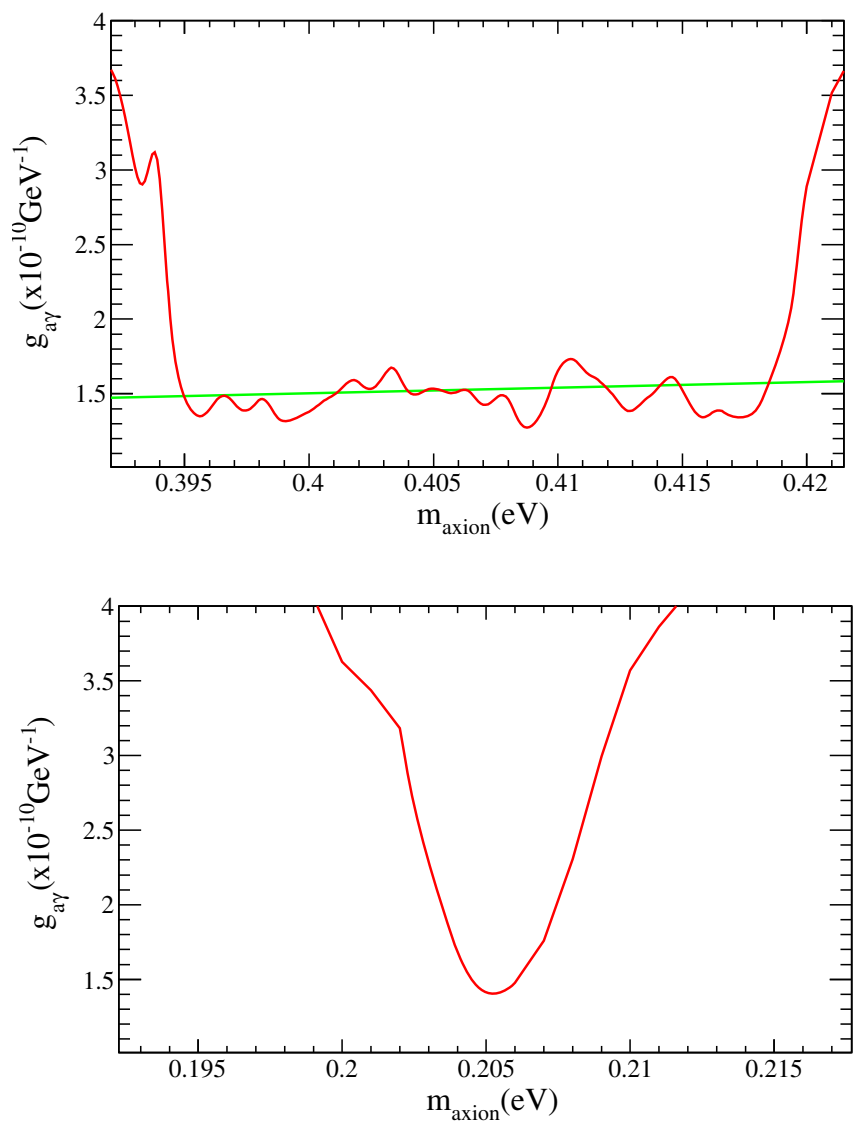

FIG. 3 (color online). Expanded view of the limit achieved during the 2012 CAST data-taking campaign, using ${ }^{4} \mathrm{He}$ as buffer gas. The plot on the bottom corresponds to the "candidate setting" at $0.2 \mathrm{eV}$, while the plot on the top is the excluded region above $0.4 \mathrm{eV}$. The green line represents the KSVZ benchmark model with $E / N=0$.

\section{CONCLUSIONS}

After finishing its initial mission of searching for solar axions up to the largest $m_{a}$ value that could be reasonably reached with ${ }^{3} \mathrm{He}$, CAST has embarked on a program of technical improvements aimed at increasing its sensitivity. Here we reported the first results from a new ${ }^{4} \mathrm{He}$ run with significantly reduced background rates in two narrow $m_{a}$ bands around 0.2 and $0.4 \mathrm{eV}$. The bounds on $g_{a \gamma}$ were significantly improved in these regions, allowing us to cross the KSVZ axion line near the highest possible ${ }^{4} \mathrm{He}$ pressure.

Since 2013, we have returned to vacuum measurements, i.e., the low-mass regime, $m_{a}<0.02 \mathrm{eV}$. With various further improvements, notably an additional $\mathrm{x}$-ray telescope, we aim at a sensitivity for $g_{a \gamma} \simeq 0.6 \times 10^{-10} \mathrm{GeV}^{-1}$ for these low masses that could even surpass a recently improved stellar-evolution bound from the helium-burning lifetime of globular-cluster stars [51]. This low-mass regime, while far away from the "axion line," is important in the context of ALPs; e.g., the propagation of $\mathrm{TeV}$ gamma rays in large-scale astrophysical magnetic fields can be addressed by a possible photon-ALP conversion.

\section{ACKNOWLEDGMENTS}

We thank CERN for hosting CAST and for technical support to operate the magnet and cryogenics. We thank the CERN CFD team for their essential contribution to the CFD work. We acknowledge support from NSERC (Canada), MSES (Croatia), CEA (France), BMBF (Germany) under the Grants No. 05 CC2EEA/9 and No. 05 CC1RD1/0 and DFG (Germany) under Grants No. HO 1400/7-1 and No. EXC-153, GSRT (Greece), NSRF: Heracleitus II, RFFR (Russia), and the Spanish Ministry of Economy and Competitiveness (MINECO) under Grants No. FPA2008-03456, No. FPA2011-24058 and No. EIC-CERN-2011-0006. This work was partially funded by the European Regional Development Fund (ERDF/FEDER), the European Research Council (ERC) under Grant No. ERC-2009-StG-240054 (T-REX), Turkish Atomic Energy Authority (TAEK), National Science Foundation (USA) under Award No. 0239812 and NASA under the Grant No. NAG5-10842. Part of this work was performed under the auspices of the U.S. Department of Energy by Lawrence Livermore National Laboratory under Contract No. DE-AC52-07NA27344.
[1] J. Jaeckel and A. Ringwald, Annu. Rev. Nucl. Part. Sci. 60, 405 (2010).

[2] A. Ringwald, Phys. Dark Univ. 1, 116 (2012).

[3] J. L. Hewett et al., arXiv:1205.2671.

[4] K. Baker et al., Ann. Phys. (Berlin) 525, A93 (2013).

[5] R. Essig et al., arXiv:1311.0029.

[6] A. Ringwald, L. J. Rosenberg, and G. Rybka, Axions and other similar particles, in K. A. Olive et al. (Particle Data Group), Chin. Phys. C 38, 090001 (2014).
[7] R. D. Peccei, Lect. Notes Phys. 741, 3 (2008).

[8] J. E. Kim and G. Carosi, Rev. Mod. Phys. 82, 557 (2010).

[9] P. Sikivie, Lect. Notes Phys. 741, 19 (2008).

[10] S. J. Asztalos et al. (ADMX Collaboration), Phys. Rev. Lett. 104, 041301 (2010).

[11] S. J. Asztalos et al. (ADMX Collaboration), Nucl. Instrum. Methods Phys. Res., Sect. A 656, 39 (2011).

[12] K. van Bibber and G. Carosi, arXiv:1304.7803.

[13] G. Rybka, Phys. Dark Univ. 4, 14 (2014). 
[14] A. Cho, Science 342, 552 (2013).

[15] O. K. Baker, M. Betz, F. Caspers, J. Jaeckel, A. Lindner, A. Ringwald, Y. Semertzidis, P. Sikivie, and K. Zioutas, Phys. Rev. D 85, 035018 (2012).

[16] D. Horns, J. Jaeckel, A. Lindner, A. Lobanov, J. Redondo, and A. Ringwald, J. Cosmol. Astropart. Phys. 04 (2013) 016.

[17] J. Jaeckel and J. Redondo, J. Cosmol. Astropart. Phys. 11 (2013) 016.

[18] J. Jaeckel and J. Redondo, Phys. Rev. D 88, 115002 (2013).

[19] D. Horns, A. Lindner, A. Lobanov, and A. Ringwald, arXiv:1309.4170.

[20] P. Sikivie, N. Sullivan, and D. B. Tanner, Phys. Rev. Lett. 112, 131301 (2014).

[21] G. Rybka and A. Wagner, Phys. Rev. D 91, 011701 (2015).

[22] The Center for Axion and Precision Physics Research (CAPP) of the Institute for Basic Science (IBS) in Korea has been dedicated to these physics goals; see http://capp.ibs .re.kr/html/capp_en/

[23] S. Neff (CAST Collaboration), Report No. CERN-SPSC2014-036; Report No. SPSC-SR-149, https://cds.cern.ch/ record/1956327.

[24] J. E. Moody and F. Wilczek, Phys. Rev. D 30, 130 (1984).

[25] G. Raffelt, Phys. Rev. D 86, 015001 (2012).

[26] E. G. Adelberger and T. A. Wagner, Phys. Rev. D 88, 031101 (2013).

[27] T. M. Leslie and J. C. Long, Phys. Rev. D 89, 114022 (2014).

[28] P. W. Graham and S. Rajendran, Phys. Rev. D 84, 055013 (2011).

[29] P. W. Graham and S. Rajendran, Phys. Rev. D 88, 035023 (2013).

[30] D. Budker, P. W. Graham, M. Ledbetter, S. Rajendran, and A. Sushkov, Phys. Rev. X 4, 021030 (2014).

[31] P. Sikivie, Phys. Rev. Lett. 51, 1415 (1983); 52, 695(E) (1984).

[32] K. Zioutas et al. (CAST Collaboration), Phys. Rev. Lett. 94, 121301 (2005).

[33] S. Andriamonje et al. (CAST Collaboration), J. Cosmol. Astropart. Phys. 04 (2007) 010.

[34] E. Arik et al. (CAST Collaboration), J. Cosmol. Astropart. Phys. 02 (2009) 008.

[35] M. Arik et al. (CAST Collaboration), Phys. Rev. Lett. 107, 261302 (2011).

[36] M. Arik et al. (CAST Collaboration), Phys. Rev. Lett. 112, 091302 (2014).

[37] D. M. Lazarus, G. C. Smith, R. Cameron, A. C. Melissinos, G. Ruoso, Y. K. Semertzidis, and F. A. Nezrick, Phys. Rev. Lett. 69, 2333 (1992).
[38] S. Moriyama, M. Minowa, T. Namba, Y. Inoue, Y. Takasu, and A. Yamamoto, Phys. Lett. B 434, 147 (1998).

[39] Y. Inoue, T. Namba, S. Moriyama, M. Minowa, Y. Takasu, T. Horiuchi, and A. Yamamoto, Phys. Lett. B 536, 18 (2002).

[40] Y. Inoue, Y. Akimoto, R. Ohta, T. Mizumoto, A. Yamamoto, and M. Minowa, Phys. Lett. B 668, 93 (2008).

[41] K. van Bibber, P. M. McIntyre, D. E. Morris, and G. G. Raffelt, Phys. Rev. D 39, 2089 (1989).

[42] S. Hannestad, A. Mirizzi, and G. Raffelt, J. Cosmol. Astropart. Phys. 07 (2005) 002.

[43] S. Hannestad, A. Mirizzi, G. G. Raffelt, and Y. Y. Y. Wong, J. Cosmol. Astropart. Phys. 08 (2007) 015.

[44] A. Melchiorri, O. Mena, and A. Slosar, Phys. Rev. D 76, 041303 (2007).

[45] S. Hannestad, A. Mirizzi, G. G. Raffelt, and Y. Y. Y. Wong, J. Cosmol. Astropart. Phys. 04 (2008) 019.

[46] S. Hannestad, A. Mirizzi, G. G. Raffelt, and Y. Y. Y. Wong, J. Cosmol. Astropart. Phys. 08 (2010) 001.

[47] M. Archidiacono, S. Hannestad, A. Mirizzi, G. Raffelt, and Y. Y. Y. Wong, J. Cosmol. Astropart. Phys. 10 (2013) 020.

[48] J. E. Kim, Phys. Rev. Lett. 43, 103 (1979).

[49] M. A. Shifman, A. I. Vainshtein, and V. I. Zakharov, Nucl. Phys. B166, 493 (1980).

[50] G. G. Raffelt, Lect. Notes Phys. 741, 51 (2008).

[51] A. Ayala, I. Domínguez, M. Giannotti, A. Mirizzi, and O. Straniero, Phys. Rev. Lett. 113, 191302 (2014).

[52] A. Friedland, M. Giannotti, and M. Wise, Phys. Rev. Lett. 110, 061101 (2013).

[53] I. G. Irastorza et al., J. Cosmol. Astropart. Phys. 06 (2011) 013.

[54] I. Shilon, A. Dudarev, H. Silva, and H. H. J. ten Kate, IEEE Trans. Appl. Supercond. 23, 4500604 (2013).

[55] E. Armengaud et al., J. Instrum. 9, T05002 (2014).

[56] R. Bähre et al., J. Instrum. 8, T09001 (2013).See also the ALPS web site, http://alps.desy.de/.

[57] S. Aune et al., J. Instrum. 9, P01001 (2014).

[58] P. Abbon et al., New J. Phys. 9, 170 (2007).

[59] S. Aune et al. (CAST Collaboration), Nucl. Instrum. Methods Phys. Res., Sect. A 604, 15 (2009).

[60] S. Andriamonje et al., J. Instrum. 5, P02001 (2010).

[61] J. Galán et al., J. Instrum. 5, P01009 (2010).

[62] M. Kuster et al., New J. Phys. 9, 169 (2007).

[63] S. Cebrián et al., Astropart. Phys. 34, 354 (2011).

[64] J. A. García et al., J. Phys. Conf. Ser. 460, 012003 (2013).

[65] A. Tomás, Ph.D. thesis, University of Zaragoza, Spain, 2013, https://cds.cern.ch/record/1557181.

[66] S. Aune et al., J. Instrum. 8, C12042 (2013).

[67] A. Tomás et al., Phys. Procedia 37, 478 (2012).

[68] D. Calvet et al., IEEE Trans. Nucl. Sci. 55, 2592 (2008). 